\title{
Computational Sustainability
}

\author{
Carla P. Gomes \\ Cornell University \\ Ithaca, NY, USA \\ gomes@cs.cornell.edu
}

\begin{abstract}
Computational sustainability [1] is a new interdisciplinary research field with the overall goal of developing computational models, methods, and tools to help manage the balance between environmental, economic, and societal needs for sustainable development. The notion of sustainable development - development that meets the needs of the present without compromising the ability of future generations to meet their needs - was introduced in Our Common Future, the seminal report of the United Nations World Commission on Environment and Development, published in 1987. In this talk I will provide an overview of computational sustainability, with examples ranging from wildlife conservation and biodiversity, to poverty mitigation, to large-scale deployment and management of renewable energy sources. I will highlight overarching computational challenges at the intersection of constraint reasoning, optimization, data mining, and dynamical systems. Finally I will discuss the need for a new approach that views computational sustainability problems as "natural" phenomena, amenable to a scientific methodology, in which principled experimentation, to explore problem parameter spaces and hidden problem structure, plays as prominent a role as formal analysis.
\end{abstract}

Acknowledgments. The author is the lead Principal Investigator of an Expedition in Computing grant on Computational Sustainability from the National Science Foundation (NSF award number: 0832782). The author thanks NSF for the research support and the grant team members for their many contributions towards the development of a vision for computational sustainability, in particular, Chris Barrett, Antonio Bento, Jon Conrad, Tom Dietterich, John Gunckenheimer, John Hopcroft, Ashish Sabharwhal, Bart Selman, David Shmoys, Steve Strogatz, and Mary Lou Zeeman.

\section{Reference}

[1] Gomes, C.P.: Computational Sustainability: Computational methods for a sustainable environment, economy, and society. The Bridge, National Academy of Engineering 39(4) (Winter 2009) 